\title{
EAl Endorsed Transactions

\section{Solving the Problem of Balancing and Complete Compensation of Reactive Power for a Three-Phase Power Supply System}

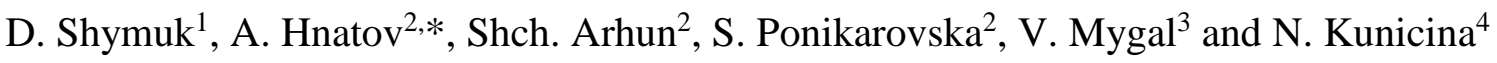 \\ ${ }^{1}$ Ivan Kozhedub Kharkiv University of Air Force, 77/79, Sumska street, Kharkiv, Ukraine 61023 \\ ${ }^{2}$ Kharkiv National Automobile and Highway University (KhNAHU), 25, Yaroslav Mudryi street, Kharkiv, Ukraine 61002 \\ ${ }^{3}$ Kharkiv Petro Vasylenko National Technical University of Agriculture, 44, Alchevskyh, Kharkiv, Ukraine 61002 \\ ${ }^{4}$ Riga Technical University, 1 Kalku Street, Riga, Latvia, LV-1658
}

\section{Abstract}

The paper deals with the development and study of the method to determine the parameters of the balancing devices of three-phase power supply systems, based on the analysis of the system operation mode with the subsequent graphoanalytical determination of the parameters of balancing and full compensation of reactive power. Complex methods of analysis of linear electrical circuits are used. The proposed method allows obtaining in a graphoanalytical way a numerical solution to the problem of balancing currents in a line and compensation of reactive power in a three-phase power supply system with an asymmetric load. The calculation results show that the application of the proposed method initiates significant balancing of currents in the lines of a three-phase power supply system. The unbalance coefficient in the reverse sequence decreased from $32.9 \%$ to $1.25 \%$. The phase difference between the voltage vectors of the phase EMF and currents does not exceed 0.7 electrical degrees.

Keywords: Smart grid, Smart homes, Smart cities, Energy, Intelligent Systems, online monitoring, diagnostics, protection, training systems, electric power industry, Energy systems control.

Received on 29 November 2019, accepted on 04 February 2020, published on 06 February 2020

Copyright (C) 2020 D. Shymuk et al., licensed to EAI. This is an open access article distributed under the terms of the Creative Commons Attribution licence (http://creativecommons.org/licenses/by/3.0/), which permits unlimited use, distribution and reproduction in any medium so long as the original work is properly cited.

doi: 10.4108/eai.13-7-2018.163093

"Corresponding author. Email: kalifus76@gmail.com

\section{Introduction}

Using electric motors as consumers of power supply systems of various purposes is the factor of increasing specific level of reactive power produced in generators and circulating in power grids. Excessive reactive power causes such negative consequences as losses in generators and lines, voltage drop in lines, lower quality of electricity in the grids as a whole. At the same time, with the widespread use of single-phase motor consumers there is a change in active-reactive phase loads. This phenomenon is inherent in the power supply of railways, urban electric transport, etc. Uneven loading of phases at its stochastic nature of occurrence leads to emergence of overcurrents and increase of costs in the grid. Therefore, balancing of currents and reduction of the reactive power circulation in the grid is an important task; its solution will help to provide the required quality of electrical energy.

Balancing of currents and voltages in electric threephase power supply systems is an important means of improving the quality of power supply [1-3]. It is through balancing that the values of currents in the line phases are matched, making the power sources load evenly. In addition, balancing contributes to the reduction of currents in the grids and, consequently, to the reduction of losses in the transportation of electrical energy [4]. 
In addition to balancing, in order to reduce losses when transmitting electrical energy, it is necessary to ensure transmission from the source to only the active power loading [5]. Balance-compensating devices are used to solve such problems. Therefore, there is a need to determine the parameters of the balance-compensating devices, which, given the active-reactive resistance of the lines, is quite a challenge.

In [6], the principle of balancing currents and reactive power compensation is described, based on calculated ratios linking the measured values of currents and load voltages with the conductivity parameters of a balancingcompensating device. However, here the parameters of power lines are not taken into account, which fact negatively affects the accuracy of balancing and compensation of reactive power.

Consideration of active-reactive resistance of lines, connecting ideal sources (with infinite power) and unbalanced active-reactive load is a factor that make it difficult to determine the balancing and compensation parameters [7-14].

To solve this problem, the authors of [7-14] use optimization algorithms and methods, as well as modern magnetic-pulse technologies.

In [7], the possibility of balance compensation and reactive energy compensation by LCL filters is considered. It is noted that, in the LCL filter study, only the harmonic attenuation and loss of active power are considered. The characteristics of the reactive power of the LCL filter are not taken into account. Therefore, it is possible to perform reactive power compensation by means of a proper filter design. The authors propose a multi-purpose optimal design method that includes both reactive power loss and reactive power compensation.

In [8-10], the use of modern magnetic-pulse technologies is considered, while rather capacitive blocks of condensers are used in the equipment under study. Such units, in addition to performing their direct functions, are excellent means of compensating for inductive power in the grid. I.e., for example, by replacing the hydraulic press or the machine for stamping with a magnetic-pulse analogue, it is possible to achieve more efficient fulfillment of its direct functions and compensation of reactive power in the power supply system of the enterprise.

In [1] a new method for determining the required amount of reactive power compensation for the power grid is offered. It is based on tracking the reactive power flow with the determination of compensation points. The authors claim that the application of the proposed method will allow optimizing the reactive power distribution, as well as reducing reactive power losses.

Based on the analysis of the mechanism of power loss and voltage drop in the distribution system, the authors of article [11] proposed a method of automatic control of reactive power compensation. This method aims at maintaining the power factor of the feeders from 0.98 to 1.0. A method of simulating continuous power flow is also suggested. The application of these methods should reduce power losses in the power supply system.

In [12], by means of search-optimization, the following parameters of the balance-compensating device are calculated, which minimize the criterion that takes into account the amplitudes of the reverse, zero sequence of currents and the phase of the positive sequence of current.

The authors of [13], in their study of modes of full reactive power compensation under the condition of minimizing the criterion of the quadratic ball metric of the reactive power of each power source, found that the choice of the initial values of the optimization parameters has a significant effect on the final result. It is shown that there can be two stable local optimums, which means that the solution of the optimization problem is ambiguous.

In [14], the search engine optimization is used to determine the parameters of a symmetric device, and the method is implemented on a visual model using MATLAB software.

Also, a number of special methods are used to solve the problem of load balance and reactive power compensation in the power supply system. For example, in [15], the authors consider the use of underground power cables in combination with overhead lines, with the reactive power of the overhead line being offset by power cables. For such compensation, it is proposed to divide the overhead line into $n$ equal sections, and to insert power cables between them. As a result, the inductive power of the overhead line will be offset by the capacitive power of the underground power cable. On the whole, the idea is not bad with an obvious technical solution, but it should be noted that its implementation significantly complicates the process of laying such a combined power line. This requires increased financial and time costs. In some cases, this solution is completely impossible to implement, for example, in mountainous or swampy terrain.

In [16] the problem of balancing of voltages of electric grids nodes in phase coordinates is considered. Here, there is a notable idea of calculating "injection" of reactive unbalanced current from the compensation device to the line that feeds the unbalanced non-uniform load with the reactive component, which enables to realize the mode of current balance and reactive power compensation in a three-phase grid. Thus, it was found that the need to take into account the resistance of the supply lines of unbalanced load from a balanced three-phase infinite power source causes complication of determining the parameters of balance and compensation of reactive fatigue in three-phase power supply systems. Precise determination of such parameters provides the methods of search engine optimization. But if the initial values of the mode parameters are ill-chosen, ambiguous solutions can be obtained.

Therefore, доцільним it is advisable to take into account the parameters of the power lines in the analytical determination of the balance parameters, and compensation of reactive power is advisable using the decomposition of systems for calculating the initial approximation parameters, computational mathematics 
used in [12, 13], the method of sequential approximations in [14], the idea of "injection" of the reactive unbalanced current from the compression device in line with [16].

The use of linear algebra methods provides the desired result, namely, the parameters of the balancing device, which provide a balancing mode and complete compensation of the reactive power in the three-phase power supply system considering the impact of the power lines resistance.

The purpose of the article is to develop and investigate a method for determining the parameters of a balancing device for three-phase power supply systems, based on the analysis of the existing mode of system operation with subsequent graphical and analytical determination of the parameters of balancing and complete reactive power compensation considering the impact of the power lines resistance.

To achieve this goal it is necessary:
- to analyze the existing mode of the grid operation;

- to create requirements for the parameters of the balancing device;

- to determine the scheme of the balancing device and the parameters of its elements;

- to analyze the efficiency of balancing and compensation, if necessary, to specify the parameters of the balancing device.

\section{Analyzing the grid operation mode}

Analysis of the grid operation mode means determining the currents and voltages of the branches of its circuit. Figure 1 shows an equivalent diagram of a three-phase power supply system with unbalanced loading [17].

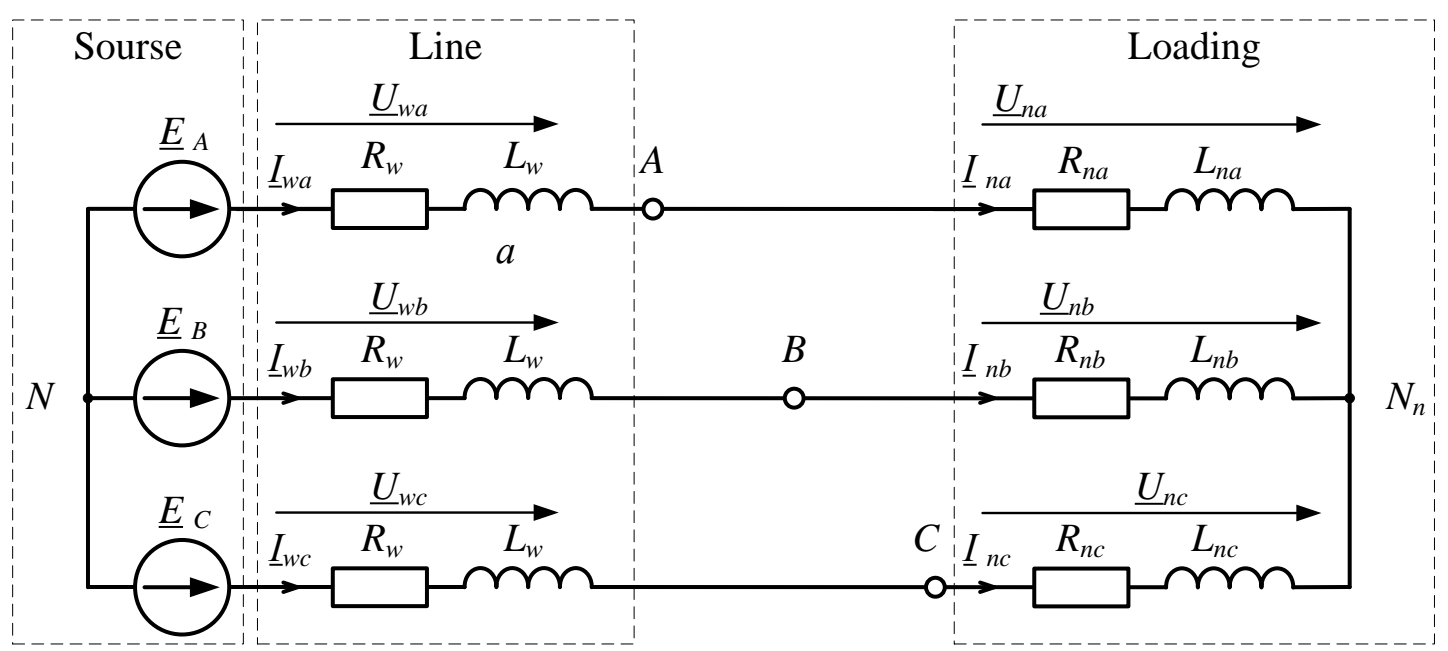

Figure 1. An equivalent diagram of a three-phase power supply system with unbalanced loading

The three-phase source of electromotive force (EMF) is balanced, of endless power with active value of harmonious voltage $E=100 \mathrm{~V}$ and frequency $50 \mathrm{~Hz}$. The line phases have the same parameters of active resistance $R_{w}=0.1 \mathrm{Ohm}$ and induction $L_{w}=0,001 \mathrm{H}$. Active resistances and inductions of branches of phase loads are the following [14]:

- $R_{n a}=1.5 \mathrm{Ohm}$,

- $R_{n b}=1.0 \mathrm{Ohm}$,

- $R_{n c}=2.0 \mathrm{Ohm}$,

- $L_{n a}=0.025 \mathrm{H}$,

- $L_{n b}=0.01 \mathrm{H}$,

- $L_{n c}=0.04 \mathrm{H}$.

The diagram calculation on Figure 1. is made in a known manner [18-21] in a complex form and presented with necessary explanations in Table 1. All calculations were carried out in the Mathcad program.

Since we consider processes under harmonic influences of the same frequency $\mathrm{f}=50 \mathrm{~Hz}$, all calculations are further carried out in complex form.

Analysis of the results of calculations given in Table 1, shows the following:

(i) The phase currents of the source and lines are equal to the phase currents of the loads.

(ii) The phase currents of the loads are unbalanced and have significant reactive components.

Quantitatively, the unbalance of the source currents is characterized by the presence of a balanced component of the negative sequence of currents, the value of the unbalance coefficient by which $\left(\mathrm{K}_{\mathrm{nc}(2)}=32,871 \%\right) \quad$ significantly exceeds the permissible value. 
Table 1. Calculation of currents in equivalent diagram in Figure 1

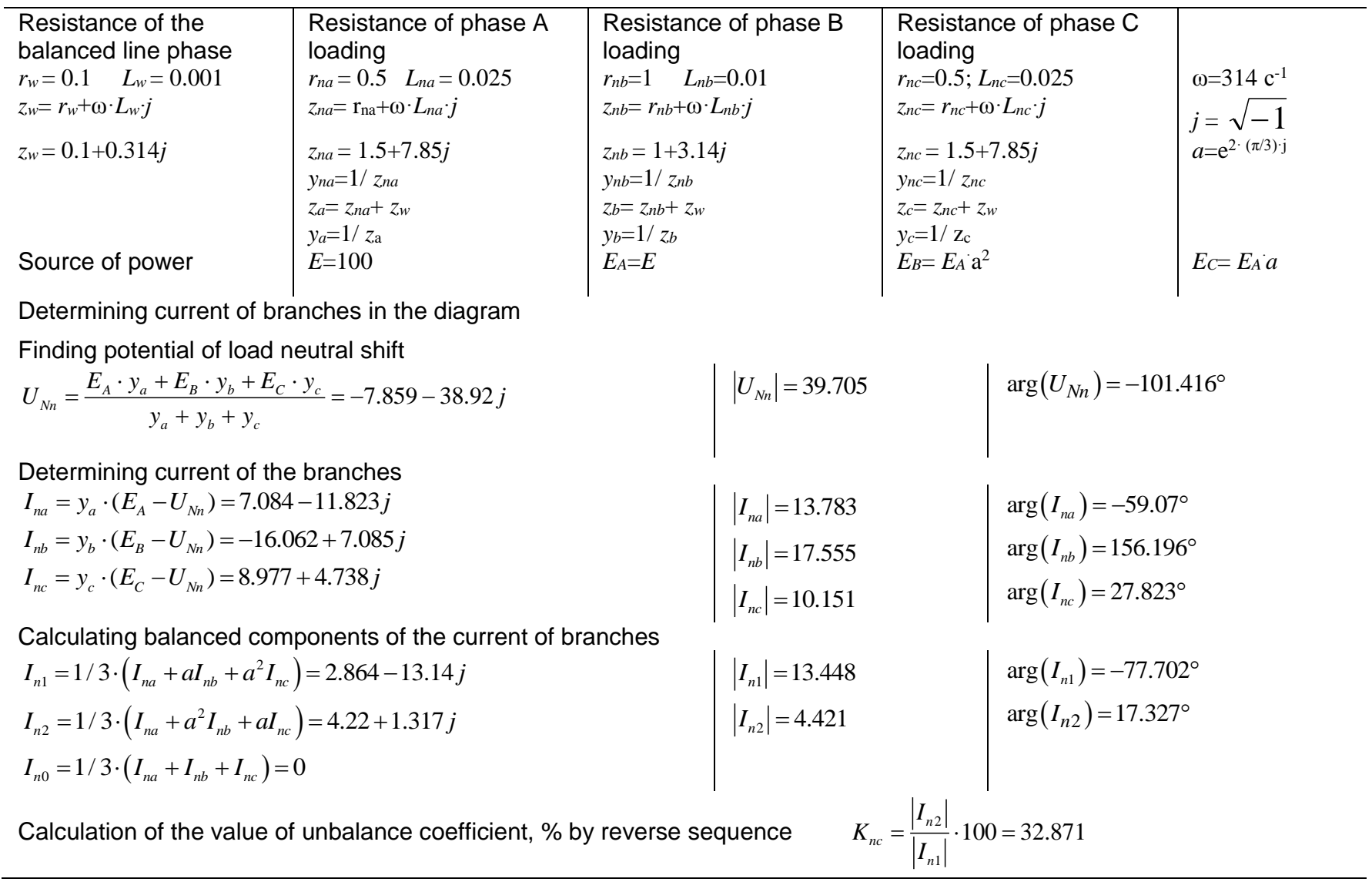

\section{Formation of requirements for the parameters of the balancing device}

In the diagram in Figure 1 the balancing device (BD) is connected to load clamps $A, B, C$ according to Figure 2 .

Requirements for the balancing device should be formulated as follows.

(i) If the purpose is to ensure only the balance of the currents of the power sources, then the BD must consume unbalanced currents that compensate for the component of the negative sequence of load currents. Then the currents of lines $\underline{I}_{w a}, \underline{I}_{w b}, \underline{I}_{w c}$ will contain only the balanced component of positive sequence $\underline{I}_{n 1} \quad$ (Table 1) from the currents of the loads considering the phase turn, meaning:

$$
\begin{gathered}
\underline{I}_{w a}=\underline{I}_{n 1}=13,448 e^{\mathrm{j}\left(-77,702^{\circ}\right)} \mathrm{A} \\
\underline{I}_{w b}=\underline{I}_{n 1} \cdot a^{2}=13,448 \mathrm{e}^{\mathrm{j}\left(162,298^{\circ}\right)} \mathrm{A} \\
\underline{I}_{w c}=\underline{I}_{n 1} \cdot a=13,448 \mathrm{e}^{\mathrm{j}\left(42,298^{\circ}\right)} \mathrm{A}
\end{gathered}
$$

By the known phase currents of the lines $\underline{I}_{w a}, \underline{I}_{w b}, \underline{I}_{w c}$ and phase currents of the load $\underline{I}_{n a}, \underline{I}_{n b}, \underline{I}_{n c}$, we calculate the phase currents $\underline{I}_{k a}, \underline{I}_{k b}, \underline{I}_{k c}$ of balancing device according the Kirchhoff first law:

$$
\begin{aligned}
& \underline{I}_{k a}=\underline{I}_{w a}-\underline{I}_{n a} \\
& \underline{I}_{k b}=\underline{I}_{w b}-\underline{I}_{n b} \\
& \underline{I}_{k c}=\underline{I}_{w c}-\underline{I}_{n c}
\end{aligned}
$$

(ii) If reactive power compensation is to be performed alongside with the balancing, the BD must compensate for both the reverse component of the load current and the reactive component of the positive sequence of load currents. Under this condition, the line current will contain only the active component of the positive sequence of load currents (Table 1):

$$
\underline{I}_{w a}=\operatorname{Re}\left(\underline{I}_{n 1}\right)=2.864 e^{\mathrm{j} 0^{\circ} \mathrm{A}}
$$




$$
\begin{gathered}
\underline{I}_{w b}=\operatorname{Re}\left(\underline{I}_{n 1}\right) \cdot a^{2}=2.864 e^{\mathrm{j} 240^{\circ}} \mathrm{A} \\
\underline{I}_{w c}=\operatorname{Re}\left(\underline{I}_{n 1}\right) \cdot a=2.864 e^{\mathrm{j} 120^{\circ}} \mathrm{A}
\end{gathered}
$$

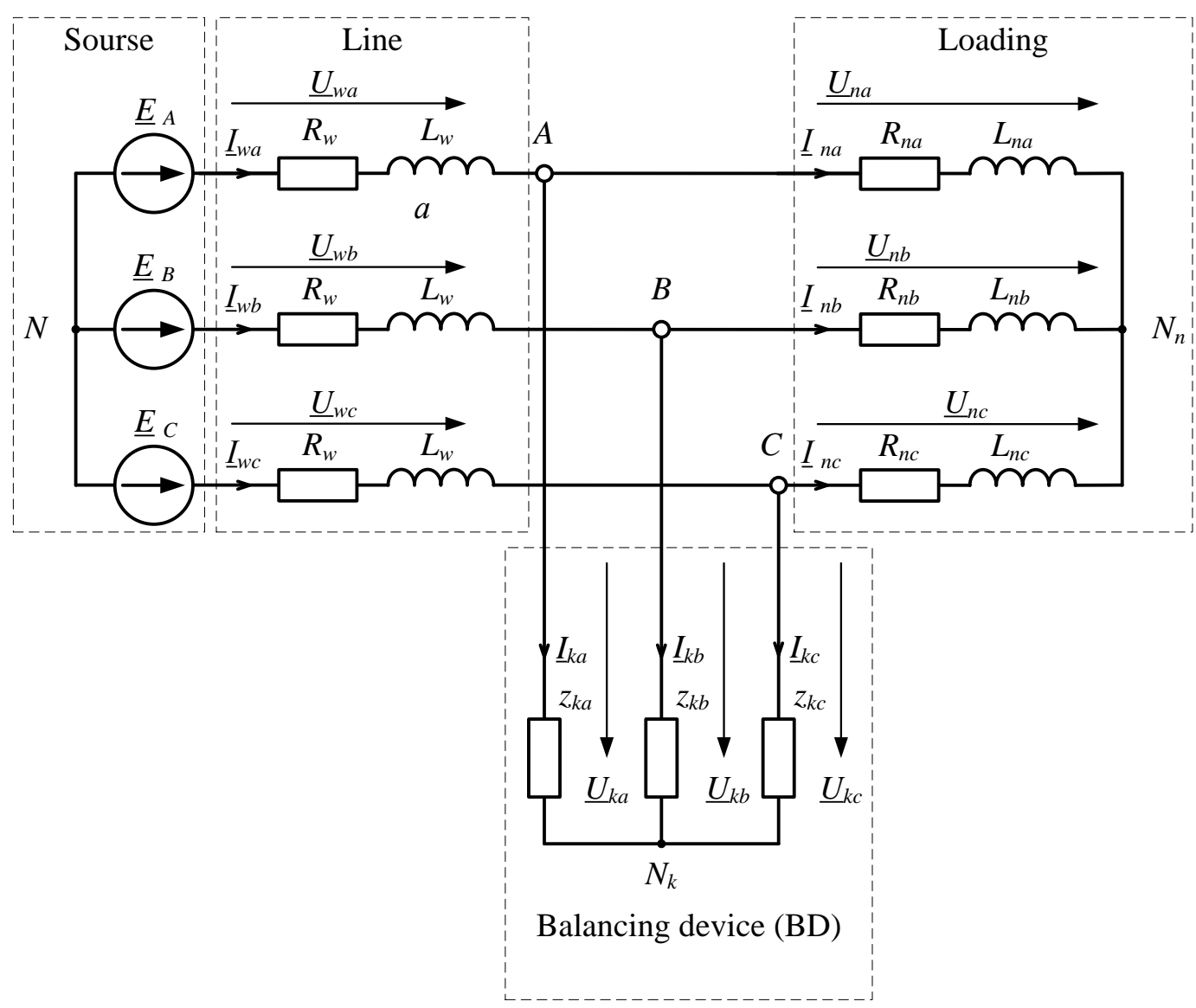

Figure 2. Equivalent diagram of three-phase system of power supply with abalanced load and connected balancing device [17]

\section{Determination the scheme of the balancing device and the parameters of its elements}

Concerning the scheme of the balancing device: we will assume that the supports are connected like a "star". If it is necessary to use the "triangle" scheme, the known resistance of the "star" can be easily calculated in the usual way.

The decisions about the type of elements are made on the basis of the following considerations: since it is impractical to increase the consumed active power, the elements of the BD must be reactive. Since there is a need to compensate for the inductive nature of the loads, the nature of the reactivity must be capacitive.

When deciding on a balancing with full reactive power compensation, the load currents should be specified for further determination of BD currents.
The balancing device currents are calculated by the formulae $(4)-(6)$. 


$$
\begin{aligned}
& \underline{U}_{n a}=\underline{E}_{A}-\underline{U}_{w a} \\
& \underline{U}_{n b}=\underline{E}_{B}-\underline{U}_{w b} \\
& \underline{U}_{n c}=\underline{E}_{C}-\underline{U}_{w c}
\end{aligned}
$$

Then we calculate the voltage of the load neutral shift

$$
\underline{U}_{N n n}=\frac{\underline{U}_{n a} \cdot y_{n a}+\underline{U}_{n b} \cdot y_{n b}+\underline{U}_{n c} \cdot y_{n c}}{y_{n a}+y_{n b}+y_{n c}}
$$

Then we specify the currents in load phases:

$$
\begin{aligned}
\underline{I}_{n n a} & =\left(\underline{U}_{n a}-\underline{U}_{N n n}\right) \cdot y_{n a} \\
\underline{I}_{n n b} & =\left(\underline{U}_{n b}-\underline{U}_{N n n}\right) \cdot y_{n b} \\
\underline{I}_{n n c} & =\left(\underline{U}_{n c}-\underline{U}_{N n n}\right) \cdot y_{n c}
\end{aligned}
$$

We calculate the phase currents of the compensator according to (4) - (6) considering condition (10) and equations $(18)-(20)$ :

$$
\begin{aligned}
\underline{I}_{k a} & =I_{1 w}-\underline{I}_{n n a} \\
\underline{I}_{k b} & =a^{2} \cdot I_{1 w}-\underline{I}_{n b} \\
\underline{I}_{k c} & =a \cdot I_{1 w}-\underline{I}_{n c}
\end{aligned}
$$

The results of calculations by formulae (10) - (23) with the necessary explanations are given in Table 2. All calculations were carried out in the Mathcad program.

Table 2 - Calculation of the balancing device currents by the diagram of Figure 2 according to formulae (10)-(23)

$I_{1 w}=\operatorname{Re}\left(I_{n 1}\right)$

Calculating voltage decrease on the lines resistances when compensated current is flowing in them

$U_{w A}=I_{1 w} \cdot z_{w}=0.286+0.899 j$

$$
\begin{aligned}
& \left|U_{w A}\right|=0.944 \\
& \left|U_{w B}\right|=0.944 \\
& \left|U_{w C}\right|=0.944
\end{aligned}
$$

$$
\begin{aligned}
& \arg \left(U_{w A}\right)=72.335^{\circ} \\
& \arg \left(U_{w B}\right)=-47.665^{\circ} \\
& \arg \left(U_{w C}\right)=-167.665^{\circ}
\end{aligned}
$$

Elaborated calculation of currents in loadings considering voltage decrease in lines phases due to the flow of compensated current
$U_{n a}=E_{A}-U_{w A}$
| $U_{n b}=E_{B}-U_{w B}$
$U_{n c}=E_{C}-U_{w C}$
$\mathrm{U}_{\mathrm{Nnn}}=\frac{\mathrm{E}_{\mathrm{na}} \cdot \mathrm{y}_{\mathrm{na}}+\mathrm{E}_{\mathrm{nd}} \cdot \mathrm{y}_{\mathrm{nb}}+\mathrm{E}_{\mathrm{nc}} \cdot \mathrm{y}_{\mathrm{nc}}}{\mathrm{y}_{\mathrm{na}}+\mathrm{y}_{\mathrm{nb}}+\mathrm{y}_{\mathrm{nc}}}=-9.292-40.736 \mathrm{j}$
$I_{n n a}=\left(U_{n a}-U_{N n n}\right) \cdot y_{n a}$
$I_{n n b}=\left(U_{n b}-U_{N n n}\right) \cdot y_{n b}$
$I_{n n b}=-16.867+7.795 j$
$I_{n n c}=\left(U_{n c}-U_{N n n}\right) \cdot y_{n c}$
$I_{n n c}=9.411+4.666 j$
Phase currents of compensator with the "star" connection circuit
$I_{k a}=I_{1 w}-I_{\text {nna }}=-4.591+12.461 j$
$I_{k b}=a^{2} \cdot I_{1 w}-I_{n n b}=15.435-10.276 j$
$\left|I_{k a}\right|=13.28$
$\left|I_{k b}\right|=18.543$
$\left|I_{k c}\right|=11.062$

$$
\begin{aligned}
& \arg \left(I_{k a}\right)=110.227^{\circ} \\
& \arg \left(I_{k b}\right)=-33.653^{\circ}
\end{aligned}
$$$$
\arg \left(I_{k c}\right)=-168.604^{\circ}
$$

To determine the phase resistances $z_{k a}, z_{k b}, z_{k c}$ of the BD it is necessary to know the corresponding vectors of phase voltages $\underline{U}_{k a}, \underline{U}_{k b}, \underline{U}_{k c}$. We know that phase voltage vectors' origin has the potential of joining points of load phases $A, B, C$ (see Figure 2) according to (14) (16), and their ends have the potential of a common point $\underline{U}_{N k}$ (Figure 2).

To determine the potential $\underline{U}_{N k}$ of the neutral point of the $\mathrm{BD} N_{k}$, we note the following.

The known parameters of phase resistances of the BD are the vectors of the phase currents of the BD. At the same time, it was previously determined that capacitors would be used as phase resistors for the BD. It is known that the vectors of voltages and capacitance currents differ from each other by the direction at angle $\pi / 2$. That is, the known parameters about each vector of the BD phase voltage are, firstly, the initial points with potentials $\underline{U}_{w a}, \underline{U}_{w b}, \underline{U}_{w c}$ according to (14) - (16) and, secondly, the directions which are perpendiculars to the current vectors $\underline{I}_{k a}, \underline{I}_{k b}, \underline{I}_{k c}$ of (18) - (20), which allows us to expressly form canonical equations of lines on a complex plane.

The potential of the intersection point of the straight lines, constructed according to the indicated signs for each phase resistance of $\mathrm{BD}$ corresponds to the potential of the common point $\underline{U}_{N k}$.

By the rules of linear algebra we know that the canonical equation of the line in the plane in the orthogonal coordinates $\mathrm{x}$, $\mathrm{y}$ has the form 


$$
A \cdot x+B \cdot y+C=0
$$

where $A, B, C$ - are constant coefficients; $x, y$ - are data points of coordinates by orthogonal axes.

For a complex plane, axis $x$ corresponds to the true axis (horizontal axis $\mathrm{Re}$ ), axis $y$ corresponds to the imaginary axis (vertical axis Im).

The values of coefficients $A_{i}, B_{i}, C_{i}$ of the canonical equations for the straight lines, on which the corresponding vectors of the stresses of the BD $\underline{U}_{k i}$ lie, where $i$ is the designation of the phases $a, b, c$ of the BD (Figure 2), are determined as follows:

$$
\begin{gathered}
A_{i}=\operatorname{Im}\left(j \cdot \underline{I}_{k i}\right) \\
B_{i}=-\operatorname{Re}\left(j \cdot \underline{I}_{k i}\right) \\
C_{i}=\operatorname{Re}\left(j \cdot \underline{I}_{k i}\right) \cdot \operatorname{Im}\left(\underline{U}_{n i}\right)-\operatorname{Im}\left(j \cdot \underline{I}_{k i}\right) \cdot \operatorname{Re}\left(\underline{U}_{n i}\right)
\end{gathered}
$$

To determine the coordinates of the intersection point of the phase voltage vectors directions, matrix equations for the coefficients of the corresponding canonical equations must be solved. For example, the coordinates of the intersection point of the directions of vectors $\underline{U}_{k a}$ and $\underline{U}_{k b}$ are determined by a column matrix $N K_{a b}$ whose elements are the real $\operatorname{Re}_{a b}$ and imaginary $\operatorname{Im}_{a b}$ of the coordinates of the point of intersection:

$$
N K_{a b}=\left(\begin{array}{ll}
\operatorname{Re}_{a b} & \operatorname{Im}_{a b}
\end{array}\right)^{T}
$$

Matrix equation to determine the coordinates of the intersection point of the directions of vectors $\underline{U}_{k a}$ and $\underline{U}_{k b}$ looks like this:

$$
N K_{a b}=A_{a b}^{-1} C_{a b}
$$

where $A_{a b}=\left(\begin{array}{ll}A_{a} & B_{a} \\ A_{b} & B_{b}\end{array}\right), C_{a b}=\left(\begin{array}{l}-C_{a} \\ -C_{b}\end{array}\right)$

The elements of matrixes $A_{a b}$ and $C_{a b}$ are determined by formulae $(25)-(27)$.
In the process of determining the coordinates of the potential $\underline{U}_{N k}$ of the point $N_{k}$, a situation may occur where the coordinates of the intersection of the different pairs of vectors' directions will differ from each other.

In this case, it is advisable to take the coordinates $\operatorname{Re}\left(\underline{U}_{N k}\right)$ and $\operatorname{Im}\left(\underline{U}_{N k}\right)$ of the potentials of point $N_{k}$ as the ones corresponding to the center of gravity of the triangle, whose vertices coordinates are determined at the intersection of the corresponding pairs of lines:

$$
\begin{aligned}
\operatorname{Re}\left(\underline{U}_{N k}\right) & =\frac{\operatorname{Re}\left(\underline{U}_{N k a b}\right)+\operatorname{Re}\left(\underline{U}_{N k b c}\right)+\operatorname{Re}\left(\underline{U}_{N k c a}\right)}{3} \\
\operatorname{Im}\left(\underline{U}_{N k}\right) & =\frac{\operatorname{Im}\left(\underline{U}_{N k a b}\right)+\operatorname{Im}\left(\underline{U}_{N k b c}\right)+\operatorname{Im}\left(\underline{U}_{N k c a}\right)}{3}
\end{aligned}
$$

After determining the coordinates of potential $\underline{U}_{N k}$ of the BD neutral by $(30,31)$ we can determine the vectors of phase voltages $\underline{U}_{k a}, \underline{U}_{k b}, \underline{U}_{k c}$ :

$$
\begin{aligned}
& \underline{U}_{k a}=\underline{U}_{n a}-\underline{U}_{N k} \\
& \underline{U}_{k b}=\underline{U}_{n b}-\underline{U}_{N k} \\
& \underline{U}_{k c}=\underline{U}_{n c}-\underline{U}_{N k}
\end{aligned}
$$

Let us determine values of $z_{k a}, z_{k b}, z_{k c}$ of capacitive resistances of $\mathrm{BD}$ :

$$
\begin{aligned}
& z_{k a}=\frac{\left|\underline{U}_{k a}\right|}{\left|\underline{I}_{k a}\right| \cdot j} ; \\
& z_{k b}=\frac{\left|\underline{U}_{k b}\right|}{\left|\underline{I}_{k b}\right| \cdot j} \underline{U}_{k a} ; \\
& z_{k c}=\frac{\left|\underline{U}_{k c}\right|}{\left|\underline{I}_{k c}\right| \cdot j}
\end{aligned}
$$

The results of calculations by formulae (24) - (35) with the necessary explanations are given in Table 3. All calculations were carried out in the Mathcad program.

Table 3. Diagram-analytical Mathcad-calculation of potential of a neutral point and phase resistances of the balancing device in Figure 2 according to formulae (24) - (35)

Coefficients of equation of voltage vector of phase $\mathrm{A}$ of a balancing device in canonic form $\left(\mathrm{A}_{\left.\mathrm{AX}+\mathrm{BAY}+\mathrm{C}_{\mathrm{A}}=0\right)}\right.$
\begin{tabular}{l|l}
$A_{A}=\operatorname{Im}\left(j \cdot I_{k a}\right)$ \\
$A_{A}=-4.591$
\end{tabular} \mid $\begin{aligned} & B_{A}=-\operatorname{Re}\left(j \cdot I_{k a}\right) \\
& B_{A}=12.461\end{aligned}$

Coefficients of equation of voltage vector of phase $B$ of a balancing device in canonic form $\left(\mathrm{A}_{\mathrm{B} X}+\mathrm{B}_{\mathrm{B}} \mathrm{y}+\mathrm{C}_{\mathrm{B}}=0\right)$
$A_{B}=\operatorname{Im}\left(j \cdot I_{k b}\right)$
$B_{B}=-\operatorname{Re}\left(j \cdot I_{k b}\right)$
$B_{B}=-10.276$
$C_{B}=\operatorname{Re}\left(j \cdot I_{k b}\right) \cdot \operatorname{Im}\left(U_{n b}\right)-\operatorname{Im}\left(j \cdot I_{k b}\right) \cdot \operatorname{Re}\left(U_{n b}\right)$
$C_{B}=-101.165$
$A_{B}=15.435$ 
Coefficients of equation of voltage vector of phase $\mathrm{C}$ of a balancing device in canonic form $\left(\mathrm{Acx}+\mathrm{Bcy}_{\mathrm{c}}+\mathrm{C}_{\mathrm{c}}=0\right)$

$A_{C}=\operatorname{Im}\left(j \cdot I_{k c}\right)$

$$
\begin{array}{l|l}
B_{C}=-\operatorname{Re}\left(j \cdot I_{k c}\right) & C_{C}=\operatorname{Re}\left(j \cdot I_{k c}\right) \cdot \operatorname{Im}\left(U_{n c}\right)-\operatorname{Im}\left(j \cdot I_{k c}\right) \cdot \operatorname{Re}\left(U_{n c}\right) \\
B_{C}=-2.186 & C_{C}=-342.463
\end{array}
$$

$A_{C}=-10.844$

Finding intersection points of voltages vectors of BD capacity separately for phases a\&b, b\&c, c\&a

$$
\begin{aligned}
A a b=\left(\begin{array}{ll}
A_{A} & B_{A} \\
A_{B} & B_{A}
\end{array}\right) ; \quad C a b=\left(\begin{array}{l}
-C_{A} \\
-C_{B}
\end{array}\right) ; \quad A b c=\left(\begin{array}{ll}
A_{B} & B_{B} \\
A_{C} & B_{C}
\end{array}\right) ; \quad C b c=\left(\begin{array}{l}
-C_{B} \\
-C_{C}
\end{array}\right) \quad A c a=\left(\begin{array}{ll}
A_{C} & B_{C} \\
A_{A} & B_{A}
\end{array}\right) ; \quad C c a=\left(\begin{array}{l}
-C_{C} \\
-C_{A}
\end{array}\right) \\
N K_{a b}=A a b^{-1} \cdot C a b=\left(\begin{array}{l}
-24.518 \\
-46.673
\end{array}\right) \quad \mid N K_{b c}=A b c^{-1} \cdot C b c=\left(\begin{array}{l}
-22.719 \\
-43.971
\end{array}\right) \quad N K_{c a}=A c a^{-1} \cdot C c a=\left(\begin{array}{c}
-22.336 \\
-45.87
\end{array}\right)
\end{aligned}
$$

The results of calculations show that coordinates of the intersection points do not coincide. Thus, we make the decision about the position of the BD neutral potential in the centre of gravity of a triangle with vertices in the points of intersection of the phase voltages pairs

$$
N_{K}=\frac{N K_{a b 0}+N K_{b c 0}+N K_{c a 0}}{3}+\frac{N K_{a b 1}+N K_{b c 1}+N K_{c a 1}}{3} \cdot j=-23.191-45.505 j
$$

Making formulae for determination of vectors of the compensator phase voltages

$$
U_{k a N k}=U_{n a}-N_{k}=122.905+44.605 j \quad U_{k b B N k}=U_{n b}-N_{k}=-27.445-40.4 j \quad U_{k c B N k}=U_{n c}-N_{k}=-25.887+132.309 j
$$

Calculated values of phase resistances of the compensator

$$
\mathrm{z}_{k a}=\frac{\left|U_{k a N k}\right|}{\left|I_{k a}\right| \cdot j}=-9.845 j \quad\left|z_{k b}=\frac{\left|U_{k b B N k}\right|}{\left|I_{k b}\right| \cdot j}=-2.634 j \quad\right| z_{k c}=\frac{\left|U_{k c N k}\right|}{\left|I_{k c}\right| \cdot j}=-12.188 j
$$

\section{Analyzing efficiency of balancing}

To analyze the efficiency of balancing it is necessary to determine the currents in the branches of the diagram in Figure 2, taking into account the values of BD resistances determined by expression (35).

The scheme in Figure 2 has nine branches, six nodes, four independent circuits. The accepted positive directions of currents of branches are marked in Figure 2, the positive voltage directions of the diagram elements coincide with the positive current directions.

According to Kirchhoff's laws for the scheme in Figure 2 the system of equations will contain four equations under the second Kirchhoff law for circuits and five equations by the first Kirchhoff law for nodes $A, B$, $C, N_{n}, N_{k}$.

The elements of the first circuit and the direction of their circumvention are as follows: $E_{a}, z_{w a}, z_{k a}, z_{k b}, z_{w b}, E_{b}$.

The elements of the second circuit and the direction of their circumvention are as follows: $E_{b}, z_{w b}, z_{k b}, z_{k c}, z_{w c}, E_{c}$.

The elements of the third circuit and the direction of their circumvention are as follows: $E_{a}, z_{w a}, z_{n a}, z_{n b}, z_{w b}, E_{b}$.

The elements of the fourth circuit and the direction of their circumvention are as follows: $E_{b}, z_{w b}, z_{n b}, z_{n c}, z_{w c}, E_{c}$.

We form a vector column of $I K$ currents of branches:

$$
I K=\left(\begin{array}{lllllllll}
\underline{I}_{w a} & \underline{I}_{w b} & \underline{I}_{w c} & \underline{I}_{k a} & \underline{I}_{k b} & \underline{I}_{k c} & \underline{I}_{n a} & \underline{I}_{n b} & \underline{I}_{n c}
\end{array}\right)^{T}
$$

Considering (36) the own matrix $\mathrm{F}$ of the diagram in Figure 2 looks like:

$$
F=\left(\begin{array}{ccccccccc}
z_{w} & -z_{w} & 0 & z_{k a} & -z_{k b} & 0 & 0 & 0 & 0 \\
0 & z_{w} & -z_{w} & 0 & z_{k b} & -z_{k c} & 0 & 0 & 0 \\
z_{w} & -z_{w} & 0 & 0 & 0 & 0 & z_{n a} & -z_{n b} & 0 \\
0 & z_{w} & -z_{w} & 0 & 0 & 0 & 0 & z_{n b} & -z_{n c} \\
1 & 0 & 0 & -1 & 0 & 0 & -1 & 0 & 0 \\
0 & 1 & 0 & 0 & -1 & 0 & 0 & -1 & 0 \\
0 & 0 & 1 & 0 & 0 & -1 & 0 & 0 & -1 \\
0 & 0 & 0 & 1 & 1 & 1 & 0 & 0 & 0 \\
0 & 0 & 0 & 0 & 0 & 0 & 1 & 1 & 1
\end{array}\right)
$$

The column-vector of input impacts $E$ relative to (36), (37) is as follows:

$$
E=\left(\begin{array}{lllllllll}
\underline{E}_{a b} & \underline{E}_{b c} & \underline{E}_{a b} & \underline{E}_{b c} & 0 & 0 & 0 & 0 & 0
\end{array}\right)^{T}
$$

where $\underline{E}_{a b}=\underline{E}_{a}-\underline{E}_{b}, \underline{E}_{b c}=\underline{E}_{b}-\underline{E}_{c}$.

We obtain vector $I K$ of complex values of currents in the result of solving the matrix equation:

$$
I K=F^{-1} E
$$

The result of the solution of the matrix equation (39) is given in Table 4.

In Table 4 we will pay attention to the values of $I_{w a}, I_{w b}$, $I_{w c}$ phase currents of the lines: we can see that by the taken measures we were able to significantly symmetrize the currents of the lines in comparison with the initial results of $I_{w a}, I_{w b}, I_{w c}$ (Table 1). Thus, the coefficient of unbalance in 
the negative sequence decreased from the initial value of $32.9 \%$ (Table 1) to the level of $1.25 \%$ (Table 4), the phase difference between the vectors of phase EMF voltages and currents does not exceed 0.7 electric degrees, which gives a reason to think that the measures taken for balancing and reactive power compensation are mostly effective.

Table 4. Calculation and assessment of coefficient of lines currents unbalance with the parameters of initial compensation

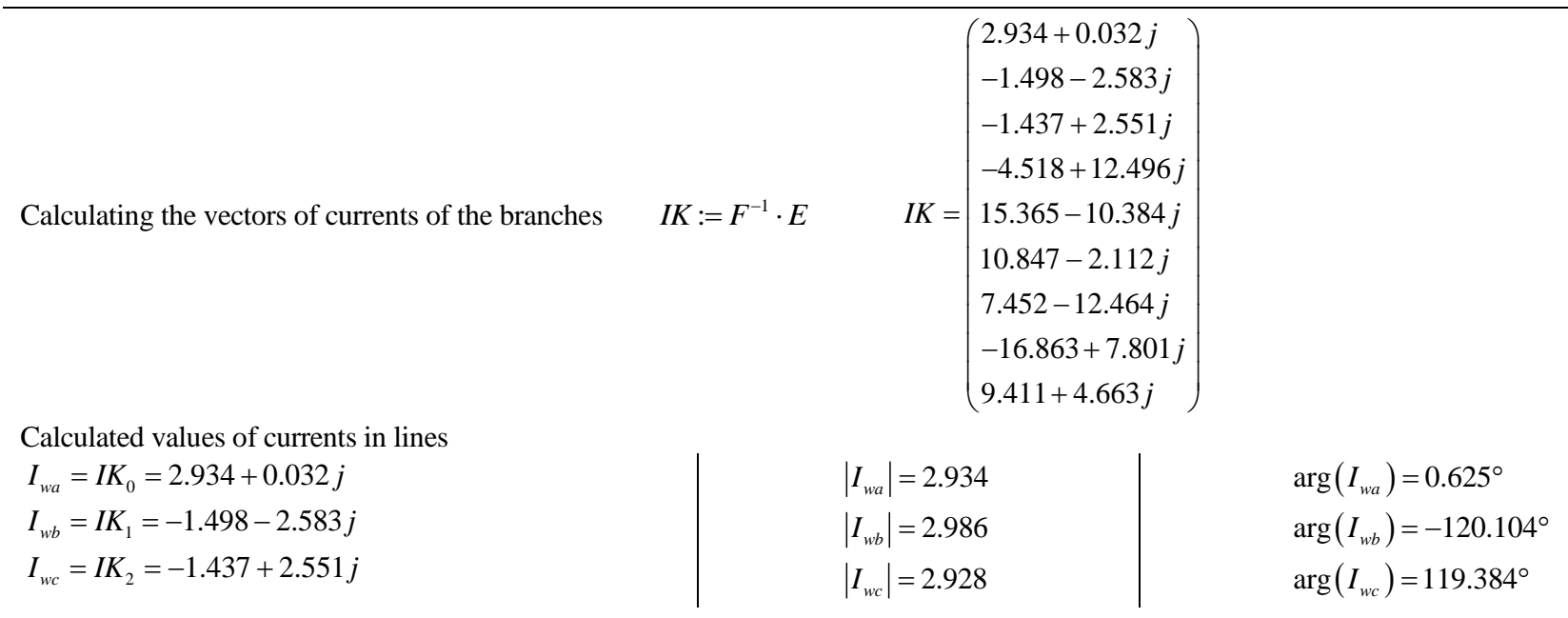

Balanced components of current in the line

$$
\begin{array}{ll}
I_{w 1}=1 / 3 \cdot\left(I_{w a}+a \cdot I_{w b}+a^{2} \cdot I_{w c}\right)=2.949-1.638 j \times 10^{-3} & I_{w 2}=1 / 3 \cdot\left(I_{w a}+a^{2} \cdot I_{w b}+a \cdot I_{w c}\right)=-0.015+0.034 j \\
\text { Coefficient of unbalance }(\%) \text { of compensated current in the line } & K_{n c 2}=\frac{\left|I_{w 2}\right|}{\left|I_{w 1}\right|} \cdot 100=1.25
\end{array}
$$

\section{Elaboration of parameters of balancing device}

The reason for the incomplete balancing is obviously the inaccurate calculation of the values of the compensating resistance of $\mathrm{BD}$.

When analyzing the reasons for such inaccuracy, the following is established. After compensation and balancing, the currents of the lines contain a symmetric component of the positive sequence with the value of the active component $\operatorname{Re}\left(\underline{I}_{w 1}\right)=2.949$ A (Table 4). This value is different from the value of $I_{1 w}$ of the first approximation (10) and is the second approximation of the symmetric current value in the lines during balancing and compensation.

To specify the parameters of the balancing device it is enough to replace in the first line of Table 4 the values of the first approximation of the symmetric current in the line $I_{1 w}=2,864$ A with the values of the second approximation $I_{1 w}=\operatorname{Re}\left(\underline{I}_{w 1}\right)=2,949$ A specified by the results of the calculation (Table 4). After that the full recalculation of parameters of the diagram of balancing device and currents of the elements of the diagram by equations (11)-(39) will take place.

The results of the elaborated calculations of the parameters of the balancing device diagram by the second approximation of the symmetric current value in the lines ( $\left.I_{1 w}=\operatorname{Re}\left(\underline{I}_{w 1}\right)=2,949 \mathrm{~A}\right)$ are shown in the form of a fragment of the Mathcad-document in Table 5.

The results shown in Table 5 clearly illustrate how, due to elaboration of the parameters of the balancing device, the effect of accurate balancing of the currents in the lines with simultaneous compensation of reactive power under unbalanced loading was achieved. At the same time, the results of the current calculation completely coincide with the results obtained by the author [14].

It should be noted that Table 1 - Table 5 are the consecutive parts of a single Mathcad document, by which graphical and analytical determination of the balancing parameters and full reactive power compensation for a three-phase power supply system is practically implemented. 
Table 5. The results of calculation and assessment of coefficient of unbalance of lines currents with elaborated compensation parameters

Calculating the vectors of currents of the branches $I K=F^{-1} \cdot E \quad I K=\left(\begin{array}{l}2.949+5.969 j \times 10^{-5} \\ -1.475-2.554 j \\ -1.475+2.554 j \\ -4.503+12.462 j \\ 15.389-10.353 j \\ 10.886-2.109 j \\ 7.452-12.462 j \\ -16.864+7.799 j \\ 9.412+4.663 j\end{array}\right)$

Calculated values of currents in lines

$I_{w a}=I K_{0}=2.949+5.969 j \times 10^{5}$

$I_{w b}=I K_{1}=-1.475-2.554 j$

$$
\begin{aligned}
& \left|I_{w a}\right|=2.949 \\
& \left|I_{w b}\right|=2.949 \\
& \left|I_{w c}\right|=2.949
\end{aligned}
$$

$I_{w c}=I K_{2}=-1.475+2.554 j$

$$
\begin{aligned}
& \arg \left(I_{w a}\right)=\left(1.16 \times 10^{-3}\right)^{\circ} \\
& \arg \left(I_{w b}\right)=-120^{\circ} \\
& \arg \left(I_{w c}\right)=119.999^{\circ}
\end{aligned}
$$

Balanced components of current in the line

$$
I_{w 1}=1 / 3 \cdot\left(I_{w a}+a \cdot I_{w b}+a^{2} \cdot I_{w c}\right)=2.949-3.593 j \times 10^{-6}
$$

Coefficient of unbalance (\%) of compensated current in the line

$$
\begin{aligned}
& I_{w 2}=1 / 3 \cdot\left(I_{w a}+a^{2} \cdot I_{w b}+a \cdot I_{w c}\right)=-2.843 \times 10^{-5}+6.328 j \times 10^{-5} \\
& \text { the line } \quad K_{n c 2}=\frac{\left|I_{w 2}\right|}{\left|I_{w 1}\right|} \cdot 100=2.352 \times 10^{-3}
\end{aligned}
$$

\section{Conclusions}

By the known parameters of asymmetric activeinductive load, source and power line, the proposed method allows obtaining graphically and analytically a numerical solution of the problem of balancing currents in the line and compensation of reactive power in a threephase power supply system with unbalanced load, which, unlike the earlier methods, takes into account the impact of the power lines resistance.

It is established that the parameters of $\mathrm{BD}$, determined according to the data of the initial analysis of the operation mode of the power supply system under unbalanced loading, do not always provide sufficient effect of balancing and compensation. The results of the calculations by the taken measures show that they lead to a significant balancing of the currents in the lines. Thus, the negative sequence unbalance coefficient decreased from $32.9 \%$ to $1.25 \%$, the phase difference between the vectors of phase EMF voltages and currents does not exceed 0.7 electric degrees. Therefore, the result obtained shows that the measures taken for balancing and reactive power compensation are sufficiently effective.

For further improvement of the balancing efficiency and reactive power compensation, the $\mathrm{BD}$ parameters should be elaborated based on the primary compensation data. After the elaboration of the parameters of the balancing device, the results were obtained, which clearly illustrate the achievement of complete balancing of currents in the lines with simultaneous compensation of the reactive power under unbalanced loading.

\section{Acknowledgements.}

This work was conducted under the Scientific research "Development of the system of energy saving and electric energy generation for vehicles", 0219U100696, funded by the Ministry of Education and Science of Ukraine.

\section{Conflict of interests.}

The authors declare that there is no conflict of interests regarding the publication of this paper.

\section{References}

[1] Yang K, Gong Y, Zhang P, Liu Z (2015) A reactive power compensation method based on tracing the power flow and loss function of power system. In: 2015 5th International Conference on Electric Utility Deregulation and Restructuring and Power Technologies (DRPT). IEEE, pp 615-619

[2] Arrillaga J, Watson NR, Chen S (2000) Power System Quality Assessment | Wiley

[3] Acha E, Agelidis V, Anaya-Lara O, Miller TJE (2002) Power electronic control in electrical systems. Newnes

[4] Mestriner D, Labella A, Bonfiglio A, Benfatto I, Li J, Ye Y, Song Z (2019) ITER Reactive Power Compensation 
Systems: analysis on reactive power sharing strategies. In: 2019 IEEE International Conference on Environment and Electrical Engineering and 2019 IEEE Industrial and Commercial Power Systems Europe (EEEIC/I\&CPS Europe). IEEE, pp 1-6

[5] Hofmann W, Schlabbach J, Just W (2012) Reactive power compensation: a practical guide. John Wiley \& Sons

[6] Liu Y-W, Rau S-H, Wu C-J, Lee W-J (2017) Improvement of power quality by using advanced reactive power compensation. IEEE Transactions on Industry Applications 54:18-24

[7] Wu Z (2018) A Novel Design Method of LCL Filters for Optimal Reactive Power Compensation in Microgrids. In: 2018 IEEE International Power Electronics and Application Conference and Exposition (PEAC). IEEE, pp 1-5

[8] Gnatov A, Argun S (2015) New Method of Car Body Panel External Straightening: Tools of Method. International Journal of Vehicular Technology 2015:1-7 . https://doi.org/10.1155/2015/192958

[9] Batygin Y, Hnatov A (2012) The features of the electrical magnetic forces excitation in the magnetic pulse sheet ferromagnetic metal working. Technical Electrodynamics 1:71-77

[10] Batygin YV, Chaplygin EA, Shinderuk SA, Strelnikova VA (2018) The main inventions for technologies of the magnetic-pulsed attraction of the sheet metals. A brief review. Elektrotexnika i Elektromexanika 3:43-52

[11] Huang X, Zhang Y, Huang H (2014) Automatic reactive power control in distribution network based on Feeder Power Factor Assessment. In: 2014 IEEE PES AsiaPacific Power and Energy Engineering Conference (APPEEC). IEEE, pp 1-4

[12] Yagup VG, Yagup EV (2016) Determination of reactive power compensation mode in four-wire three-phase electric power supply system using search engine optimization. Tekhnichna Elektrodynamika 1:60-66
[13] Yagup VG, Yagup EV (2016) Investigation of modes of full compensation of reactive power in a three-phase power supply system. Elektrotexnika i Elektromexanika 2:61-65

[14] Yagup EV (2016) Simulation of an unbalanced power supply system using optimization to determine the parameters of a balancing device. Collection of scientific works of the Ukrainian State University of Railway Transport 130-138

[15] Karki U, Gunasekaran D, Peng FZ (2015) Reactive compensation of overhead AC transmission lines using underground power cables. In: 2015 IEEE Power \& Energy Society General Meeting. IEEE, pp 1-5

[16] Gnilicz'ky’j VV, Polischuk OA (2015) Development of the model of voltage equation in three-phase grids. Vostochno-Evropeyskiy zhurnal peredovyih tehnologiy 1114

[17] Shymuk D (2019) Graphoanalytic determination of symmetry parameters and total compensation of reactive power for a three-phase power supply system. Vehicle and Electronics Innovative Technologies 15:6-16

[18] Bird J (2017) Electrical circuit theory and technology. Routledge

[19] Gnatov A, Argun S, Ulyanets O (2017) Joint innovative double degree master program "energy-saving technologies in transport." In: 2017 IEEE First Ukraine Conference on Electrical and Computer Engineering (UKRCON). Kiev, Ukraine, pp 1203-1207

[20] Patlins A, Hnatov A, Arhun S (2018) Safety of Pedestrian Crossings and Additional Lighting Using Green Energy. In: Transport Means 2018: Proceedings of 22nd International Scientific Conference. Kaunas University of Technology, Lietuva, Trakai, Kaunas, pp 527-531

[21] Arhun S, Hnatov A, Dziubenko O, Ponikarovska S (2019) A Device for Converting Kinetic Energy of Press Into Electric Power as a Means of Energy Saving. Journal of the Korean Society for Precision Engineering 36:105-110. https://doi.org/10.7736/KSPE.2019.36.1.105 